\title{
Supporting Information \\ Effectiveness of resistive vibration exercise and whey protein supplementation plus alkaline salt on the skeletal muscle proteome following 21 days of bedrest in healthy males
}

\author{
Helena C. Kenny ${ }^{1,2}$, Georg Tascher ${ }^{3,5,6}$, Anna Ziemianin ${ }^{3,5}$, Floriane Rudwill ${ }^{4}$, Alexandre \\ Zahariev $^{4}$, Isabelle Chery ${ }^{4}$, Guillemette Gauquelin-Koch ${ }^{5}$, Marie-Pierre Barielle9, Martina \\ Heer $^{7,8}$, Stephane Blanc ${ }^{4}$, Donal J. O'Gorman ${ }^{1,2 *}$, Fabrice Bertile ${ }^{3 *}$
}

${ }^{1} 3 U$ Diabetes Partnership, School of Health and Human Performance, Dublin City University, Dublin, Ireland;

${ }^{2}$ National Institute for Cellular and Biotechnology, Dublin City University, Dublin, Ireland;

${ }^{3}$ Université de Strasbourg, CNRS, IPHC UMR 7178, Département Sciences Analytiques, F-67000 Strasbourg, France

${ }^{4}$ Université de Strasbourg, Institut Pluridisiplinaire Hubert Curien, Départment d'Ecologie, Physiologie et Ethologie. CNRS, UMR7178, Strasbourg, France;

${ }^{5}$ Centre National d'Etudes Spatiales (CNES), Paris, France

${ }^{6}$ Institute of Biochemistry II, Goethe University Hospital, D-60590 Frankfurt am Main, Germany

${ }^{7}$ Profil, Hellersbergstrasse 9, D-41460 Neuss, Germany

${ }^{8}$ Institute of Nutrition and Food Sciences, University of Bonn, Germany

${ }^{9}$ Institut de Médecine et de Physiologie Spatiales, Toulouse, France

*equal contribution to the work

Address for correspondence:

Dr. Donal J. O'Gorman,

3U Diabetes Consortium,

School of Health and Human Performance,

Dublin City University,

Glasnevin, Dublin 9.

Email: donal.ogorman@dcu.ie

Phone: +353(1)700-5000 
TOC for Supporting Information:

The following supporting information is available free of charge at ACS website http://pubs.acs.org

- Supplemental Table 1. Group assignment

- Supplemental Table 2. Detailed protein abundances and fold changes 
Supplemental Table 1.

Supplemental Table 1. Group assignment

\begin{tabular}{cccc}
\hline \hline Subject & Campaign 1 & Campaign 2 & Campaign 3 \\
\hline A & RVE & NEX & CONT \\
B & CONT & RVE & NEX \\
C & RVE & NEX & CONT \\
D & NEX & CONT & RVE \\
E & NEX & CONT & RVE \\
G & NEX & CONT & RVE \\
J & RVE & NEX & CONT \\
K & NEX & CONT & RVE \\
\hline$C O N T(n=8)$ & & $R V E(n=8)$ & $N E X(n=8)$
\end{tabular}

